\title{
On hermitian Pfister forms
}

\author{
N. Grenier-Boley, E. Lequeu, M. G. Mahmoudi
}

September 12, 2007

\begin{abstract}
Let $K$ be a field of characteristic different from 2. It is known that a quadratic Pfister form over $K$ is hyperbolic once it is isotropic. It is also known that the dimension of an anisotropic quadratic form over $K$ belonging to a given power of the fundamental ideal of the Witt ring of $K$ is lower bounded. In this paper, weak analogues of these two statements are proved for hermitian forms over a multiquaternion algebra with involution. Consequences for Pfister involutions are also drawn. An invariant $u_{\alpha}$ of $K$ with respect to a non-zero pure quaternion of a quaternion division algebra over $K$ is defined. Upper bounds for this invariant are provided. In particular an analogue is obtained of a result of Elman and Lam concerning the $u$-invariant of a field of level at most 2 .
\end{abstract}

\section{Introduction}

Throughout this paper, the characteristic of the base field is supposed to be different from 2. Pfister forms play a fundamental role in the theory of quadratic forms. In the literature, efforts have been made to find analogues of these forms in the framework of central simple algebras with involution or of hermitian forms over such algebras. In the first case, a notion of Pfister involution has been defined by Shapiro (see Subsection 2.4): it is nothing but a central simple algebra endowed with an orthogonal involution which is a tensor product of quaternion algebras with involution. Shapiro has also stated a conjecture which predicts a close relation between Pfister involutions and quadratic Pfister forms (see Conjecture 2.8) which has recently been proved by Becher [2]. In the second case, a notion of hermitian Pfister form over a central simple algebra with involution $(A, \sigma)$ has been defined by Lewis [10] as the hermitian form over $(A, \sigma)$ induced by a quadratic Pfister form over the subfield of the center of $A$ consisting in elements invariant under $\sigma$ (see Subsection 2.3).

In the first part of this paper, we prove weak analogues of two important properties satisfied by quadratic forms in the framework of hermitian forms over multiquaternion algebras with involution. The first of these two properties is the fact that quadratic Pfister forms are hyperbolic once they are isotropic. Our first main result is the following which will be restated later in a more detailed form (see Theorem 3.3 below):

Theorem 1.1. Let $(A, \sigma)=\left(Q_{1}, \sigma_{1}\right) \otimes_{K} \cdots \otimes_{K}\left(Q_{n}, \sigma_{n}\right)$ be a multiquaternion algebra with involution. Then there exists a non-negative integer $s<n$ which only depends on the decomposition of $(A, \sigma)$ such that for every hermitian Pfister form $h$ over $(A, \sigma), 2^{s} \times h$ is hyperbolic as soon as $h$ is isotropic. 
Several facts are recalled in Section 2 and the beginning of Section 3 is devoted to the proof of Theorem 3.3 which is a slightly more detailed statement of Theorem 1.1. Jean-Pierre Tignol pointed out to us that the existence of an integer $s(h)$ (depending on $h$ ) such that $2^{s(h)} \times h$ is hyperbolic can also be obtained using a local-global principle for hermitian forms proved by Lewis and Unger [11]. Our result provides more information about the power $s(h)$ : it states that $s(h)$ can be chosen independently of $h$ and also gives an upper bound for it.

Another important result is Arason and Pfister's Hauptsatz which gives a dimension-theoretic necessary condition for a quadratic form to belong to a given power of the fundamental ideal of the Witt ring (see Theorem 3.6). In the second part of Section 3, we prove a weak analogue of this result (see Theorem 3.7). In the last part of this Section, we focus on Pfister involutions. Among other things, we obtain a new proof of Conjecture 2.8 in the case of linked fields.

In the second part of this paper, we define a certain invariant $u_{\alpha}$ associated to $K$ for which we give upper bounds under certain hypotheses. Recall that the $u$-invariant of a field $K$ is the supremum over the dimension of all quadratic forms $\varphi$ over $K$ such that $\varphi$ is anisotropic and that the level of a field $K$, denoted by $s(K)$, is the smallest positive integer $s$ such that -1 can be written as a sum of $s$ squares in $K$. If we denote by $q$ the number of square classes in $K^{\times}$and if $K$ is a non-real field, Kneser has proved that $u(K) \leqslant q$ (see [14, Chapter 2, $\S 16])$. This result has been refined in [4] when the value of the level of $K$ is small:

Theorem 1.2 (Elman-Lam). Let $K$ be a field whose level satisfies $s(K) \leqslant 2$. Then, either $u(K)=q$ or $u(K) \leqslant q / 2$.

Given a non-zero symmetric or skew-symmetric element $\alpha$ of a quaternion division algebra with canonical involution $(Q, \gamma)$ over $K$, we define the invariant $u_{\alpha}(K)$ to be the supremum over the dimension of all quadratic forms $\varphi$ over $K$ such that $\varphi \cdot\langle\alpha\rangle$ is anisotropic over $(Q, \gamma)$. Denote by $s(K, \sigma)$ the (hermitian) level of a field $K$ with an involution $\sigma$, that is the smallest integer $s$ such that -1 is can be written as a sum of $s$ hermitian squares, i.e., elements of the form $\sigma(x) x$ where $x \in K$. We first prove an analogue of Kneser's result above for the invariant $u_{\alpha}$ (see Proposition 4.5). Our second main result is an analogue of Theorem 1.2 for the invariant $u_{\alpha}$ :

Theorem 1.3. Let $\alpha$ be a skew-symmetric element in $Q^{\times}$with respect to its canonical involution $\gamma$. Suppose that the level of $\left(K(\alpha),\left.\gamma\right|_{K(\alpha)}\right)$ is at most 2 . Then, either $u_{\alpha}(K)=\left|K^{\times} / G_{\alpha}\right|$ or $u_{\alpha}(K) \leqslant \frac{1}{2}\left|K^{\times} / G_{\alpha}\right|$.

Here $G_{\alpha}$ is the similarity group of the one-dimensional skew-hermitian form $\langle\alpha\rangle$ : $G_{\alpha}=\left\{c \in K^{\times} \mid\langle c \alpha\rangle \simeq\langle\alpha\rangle\right.$ as skew-hermitian forms over $\left.(Q, \gamma)\right\}$. These results are proved in Section 4 .

\section{Basic results and notations}

¿From now on, every central simple algebra is supposed to be finite-dimensional over its center and every module over such an algebra is supposed to be a finitely generated right module. 


\subsection{Central simple algebras}

The general reference for the theory of central simple algebras with involution is $[8]$.

Let $K$ be a field and $A$ be a central simple algebra over $K$. Suppose that $A$ is endowed with an involution $\sigma$, that is a ring antiautomorphism of $A$ of order 2. The map $\sigma$ restricts to an involution of $K$ and there are two cases: if $\left.\sigma\right|_{K}$ is the identity map, we say that $\sigma$ is of the first kind, otherwise $\left.\sigma\right|_{K}$ is of the second kind. We will always denote by $F$ the subfield of $K$ consisting in central invariant elements under $\sigma$. Then $\sigma$ is of the first kind if $F=K$ and $\sigma$ is of the second kind if $K / F$ is a quadratic field extension. If $\sigma$ is of the second kind, we will denote by - the non-trivial automorphism of $K / F$.

A field $L$ containing $K$ is called a splitting field of $A$ if $A \otimes_{K} L$ is isomorphic to a full matrix algebra over $L$. By Wedderburn's Theorem, such a field always exists (see [8, Theorem 1.1]). An involution of the first kind is said to be orthogonal (resp. symplectic) if it is adjoint to a symmetric (resp. skew-symmetric) bilinear form after scalar extension to a splitting field of $A$. An involution of the second kind is said to be unitary.

Let $(A, \sigma)$ and $(B, \tau)$ be two central simple algebras with involution over $K$. We will say that $(A, \sigma)$ and $(B, \tau)$ are isomorphic (or simply that $\sigma$ and $\tau$ are isomorphic if the algebras are clear from the context) if there exists a $K$-algebra isomorphism $f: A \simeq B$ such that $\tau \circ f=f \circ \sigma$. When $\left.\sigma\right|_{K}=\left.\tau\right|_{K}$, the tensor product of $(A, \sigma)$ and $(B, \tau)$ refers to $\left(A \otimes_{K} B, \sigma \otimes \tau\right)$ also denoted $(A, \sigma) \otimes_{K}(B, \tau)$.

A central simple algebra of degree 2 is called a quaternion algebra. As $\operatorname{char}(K) \neq 2$, any quaternion algebra $Q$ over $K$ has a quaternion basis $\{1, i, j, k\}$, that is a basis of the $K$-algebra $Q$ subject to the relations $i^{2}=a \in K^{\times}, j^{2}=$ $b \in K^{\times}, i j=k=-j i$. This algebra $Q$ is usually denoted by $(a, b)_{K}$. Every quaternion algebra has an unique involution of symplectic type (usually denoted by $\gamma$ ) called the canonical involution of $Q$. One has $\gamma(i)=-i, \gamma(j)=-j$. A tensor product of quaternion algebras with involution over $K$ is called a multiquaternion algebra with involution. Let $(A, \sigma)$ be a multiquaternion algebra with involution. Given a decomposition $(A, \sigma)=\left(Q_{1}, \sigma_{1}\right) \otimes_{K} \cdots \otimes_{K}\left(Q_{n}, \sigma_{n}\right)$ of $(A, \sigma)$ (where the $\left(Q_{i}, \sigma_{i}\right)$ 's are quaternion algebras with involution), we define an integer $r$ as follows. Take the set of all non-split quaternion algebras $Q_{i}$ appearing in this decomposition. Partition this set into equivalence classes $C_{j}$ with isomorphism as the equivalence relation. Let $\bmod C_{j}$ be the number of elements in equivalence class $C_{j}$. Then $r$ is the number of equivalence classes for which $\bmod C_{j}$ is odd.

Example 2.1. Take $n=4$.

Suppose that $Q_{1}, Q_{2}, Q_{3}, Q_{4}$ are division algebras with $Q_{1} \simeq Q_{2}$ and $Q_{3} \simeq Q_{4}$ and $Q_{1} \not Q_{3}$ then $r=0$.

Suppose that $Q_{1}, Q_{2}, Q_{3}$ are division algebras with $Q_{1} \simeq Q_{2} \simeq Q_{3}$ and $Q_{4}$ is split then $r=1$.

Suppose that $Q_{1}, Q_{2}, Q_{3}, Q_{4}$ are division algebras and they are pairwise non-isomorphic then $r=4$.

Remark 2.2. Note that two different decompositions of $(A, \sigma)$ may have different associated $r$. For instance consider the situation where $\left(Q_{1}, \sigma_{1}\right)$ and $\left(Q_{2}, \sigma_{2}\right)$ are two quaternion division algebra with involutions of the first kind over a field 
$K$ and $A=Q_{1} \otimes Q_{2}$ is of index 2. Then $(A, \sigma)=\left(Q_{1}, \sigma_{1}\right) \otimes\left(Q_{2}, \sigma_{2}\right)$ has a decomposition $(A, \sigma) \simeq\left(M_{2}(K), \tau_{1}\right) \otimes(Q, \tau)$ where $Q$ is a quaternion division algebra and $\tau_{1}$ and $\tau$ are involutions of the first kind (see also Proposition 3.1 below). For the first decomposition we have $r=2$ and for the second one we have $r=1$.

¿From now on, $(A, \sigma)$ will denote a central simple algebra with involution over a field $K$.

\subsection{The Witt group of a central simple algebra with invo- lution}

The standard reference for the theory of hermitian forms and for the Witt group of a central simple algebra is $[14$, Chapter 7,10$]$.

Let $\varepsilon= \pm 1$. An $\varepsilon$-hermitian form over $(A, \sigma)$ is a pair $(M, h)$ where $M$ is a right $A$-module and $h$ is a map $h: M \times M \rightarrow A$ which is $\sigma$-sesquilinear in the first argument, linear in the second argument and which satisfies

$$
h(y, x)=\varepsilon \sigma(h(x, y)) \text { for any } x, y \in M .
$$

If $\varepsilon=1$ (resp. -1), an $\varepsilon$-hermitian form is called a hermitian (resp. skewhermitian) form. We will always implicitly assume that the forms considered are non-degenerate. If $A=D$ is a division algebra, every hermitian form over $(D, \sigma)$ can be diagonalized and such a diagonalization will be denoted by $\left\langle a_{1}, \cdots, a_{n}\right\rangle$. Any hermitian or skew-hermitian form $(M, h)$ over $(A, \sigma)$ has an adjoint involution defined on $\operatorname{End}_{A}(M)$, see $[8, \S 4]$.

The form $h$ is said to be isotropic if there is an $x \in M \backslash\{0\}$ such that $h(x, x)=0$, anisotropic otherwise. Let $(M, h)$ and $\left(M^{\prime}, h^{\prime}\right)$ be two $\varepsilon$-hermitian forms over $(A, \sigma)$. If these forms are isometric then we write $h \simeq h^{\prime}$ for short.

The orthogonal sum induces a commutative monoïd structure on the set of isometry classes of non-degenerate $\varepsilon$-hermitian forms over $(A, \sigma)$. The Witt group of $(A, \sigma)$ is the quotient group of the Grothendieck group of this commutative monoïd by the subgroup generated by hyperbolic forms and is denoted by $W^{\varepsilon}(A, \sigma)$. In the case where $A=K$ and $\varepsilon=1$, the tensor product can be used to define a ring structure on $W(K, \sigma)$. Moreover, if $\sigma=\operatorname{Id}_{K}$, this ring is the usual Witt ring $W(K)$. The tensor product endows $W^{\varepsilon}(A, \sigma)$ with a $W\left(K,\left.\sigma\right|_{K}\right)$-module structure and hence with a $W(F)$-module structure via the usual restriction map $W(F) \rightarrow W\left(K,\left.\sigma\right|_{K}\right)$.

\subsection{Hermitian Pfister forms}

The fundamental ideal of the Witt ring $W(K)$ is denoted by $I(K)$ and, for all $n>0$, the $n$-th power of this ideal is denoted by $I^{n}(K)$. The ideal $I^{n}(K)$ is additively generated by the so-called $n$-fold Pfister forms $\left\langle\left\langle a_{1}, \cdots, a_{n}\right\rangle\right\rangle=$ $\left\langle 1, a_{1}\right\rangle \otimes \cdots \otimes\left\langle 1, a_{n}\right\rangle$. The notion of hermitian Pfister form was first defined by Lewis in $[10]$ in the following way:

Definition 2.3. An $n$-fold hermitian Pfister form (or a hermitian Pfister form) over $(A, \sigma)$ is a hermitian form $(V, h)$ over $(A, \sigma)$ such that $h \simeq \varphi \cdot\langle 1\rangle$ where $\varphi$ is an $n$-fold quadratic Pfister form over $F$. 
It is well-known that quadratic Pfister forms are hyperbolic once they are isotropic. For convenience, we introduce the following definition :

Definition 2.4. Let $h$ be an $\varepsilon$-hermitian form over $(A, \sigma)$. We say that $h$ satisfies the property $(I \Rightarrow H)$ if $h$ is hyperbolic whenever it is isotropic.

If $A=K$ (resp. a quaternion division algebra) and $\sigma=-$ (resp. the canonical involution of $A$ ), we see easily, via the usual injection of Witt groups $W(A, \sigma) \hookrightarrow W(F)$, that a hermitian Pfister form over $(A, \sigma)$ satisfies the property $(I \Rightarrow H)$. For quaternion division algebras endowed with orthogonal or unitary involutions, the answer has been given by Serhir :

Theorem 2.5 (Serhir). (1) Let $A$ be a quaternion division algebra over $K$ and let $i, j$ be two non-zero pure quaternions satisfying $j i=-i j$. Let $\sigma$ be the orthogonal involution over $A$ defined by the relations $\sigma(i)=-i$ and $\sigma(j)=j$. Then, any hermitian Pfister form over $(A, \sigma)$ satisfies the property $(I \Rightarrow H)$.

(2) Let $A$ be a quaternion division algebra and $\sigma$ be an involution of the second kind over $A$. Then, any hermitian Pfister form over $(A, \sigma)$ satisfies the property $(I \Rightarrow H)$.

Proof. See [15, Proposition 3.1] for (1), and [16, Théorème 1.4] for (2).

We leave it to the reader to verify that Theorem 2.5(1) can be restated as

Theorem 2.6. Let $h$ be a skew-hermitian form over $(Q, \gamma)$. If $h \simeq \varphi \cdot\langle\alpha\rangle$, where $\alpha \in Q^{\times}$is a pure quaternion and $\varphi$ is a quadratic Pfister form over $K$, then $h$ satisfies the property $(I \Rightarrow H)$.

Remark 2.7. Let us keep the notations of Theorem 2.6. Let $C$ be the conic associated to $Q$ and $K(C)$ be the function field of $C$. In [13, Proposition 3.3], Parimala, Sridharan and Suresh have shown that the canonical homomorphism $W^{-1}(Q, \gamma) \rightarrow W^{-1}\left(Q \otimes_{K} K(C), \gamma \otimes \operatorname{Id}_{K(C)}\right)$ is injective. Then Theorem 2.6 can also be proved using this result.

\subsection{Pfister involutions}

In $[18$, Chapter 9, (9.17)], Shapiro formulates the following conjecture:

Conjecture 2.8. Let $\left(Q_{1}, \sigma_{1}\right), \cdots,\left(Q_{n}, \sigma_{n}\right)$ be quaternion algebras over $K$ with involutions of the first kind such that $\sigma_{1} \otimes \cdots \otimes \sigma_{n}$ is orthogonal. If $Q_{1} \otimes_{K} \cdots \otimes_{K} Q_{n}$ is split, then $\sigma_{1} \otimes \cdots \otimes \sigma_{n}$ is adjoint to an $n$-fold quadratic Pfister form over $K$.

In the above conjecture, such an involution $\sigma_{1} \otimes \cdots \otimes \sigma_{n}$ is called a Pfister involution. For short, we will refer to this conjecture for $n$ quaternion algebras as $\operatorname{PC}(n)$.

Recall that a field $K$ is said to be linked if any tensor product of quaternion algebras over $K$ is Brauer-equivalent to a single quaternion algebra. Standard examples of linked fields are finite fields, local fields, global fields, fields of transcendence degree at most 2 over an algebraically closed field or fields of transcendence degree 1 over a real closed field, see [18, 9.14].

Conjecture 2.8 was proved by Shapiro for $n \leq 5$ in [18] and over number fields by Wadsworth and Shapiro in [20]. For $n \leq 4$, there is another proof due 
to Bayer-Fluckiger, Parimala and Quéguiner-Mathieu in [1]. This conjecture was also proved differently by Serhir and Tignol in [17] for $n \leq 5$. Moreover, they prove that $\mathrm{PC}(n)$ holds for all $n$ over special fields among which number fields and linked fields. Finally, Becher has recently proved this conjecture in general, see [2].

\section{Hermitian Pfister forms over multiquaternion algebras with involution}

\subsection{Preliminary results}

In this Subsection, $(A, \sigma)$ will be a biquaternion algebra with involution, that is $(A, \sigma)=\left(Q_{1}, \sigma_{1}\right) \otimes_{K}\left(Q_{2}, \sigma_{2}\right)$ where $Q_{1}, Q_{2}$ are quaternion algebras. We suppose that $A$ is not division and prove that $(A, \sigma)$ can be decomposed (as algebra with involution) in a special way.

Proposition 3.1. Suppose that the index of $A$ is 2 and that $\sigma$ is of the first kind. Then $(A, \sigma) \simeq\left(\mathrm{M}_{2}(K), \tau_{1}\right) \otimes_{K}(Q, \tau)$ where $Q$ is a quaternion division algebra. Moreover, the involution $\tau_{1}$ can be chosen of orthogonal type.

Proof. If $\sigma$ is symplectic, the result is due to Serhir and Tignol, see [17, Proposition]. Suppose now that $\sigma$ is orthogonal and let $Q$ be the quaternion division algebra Brauer-equivalent to $A$. Then $A \simeq \operatorname{End}_{Q}(V)$ where $V$ is a free $Q$-module of rank 2. We consider $Q$ endowed with its canonical involution $\gamma$ and we see $\sigma$ as an involution on $\operatorname{End}_{Q}(V)$. The involution $\sigma$ is adjoint to a skew-hermitian form $(V, h)$ over $(Q, \gamma)$. Write $h \simeq\left\langle a_{1}, a_{2}\right\rangle$. As $\sigma$ is a Pfister involution, the discriminant of $h$ is trivial hence $\operatorname{Nrd}_{Q / K}\left(a_{1}\right)=\operatorname{Nrd}_{Q / K}\left(a_{2}\right) \in$ $K^{\times} / K^{\times 2}$. By [3, Proposition 5.1], there exists $\lambda \in K^{\times}$such that $h \simeq\langle 1, \lambda\rangle .\left\langle a_{1}\right\rangle$. We obtain the desired decomposition with $\tau_{1}$ adjoint to the quadratic form $\langle 1, \lambda\rangle$ over $K$ and $\tau$ adjoint to the skew-hermitian form $\left\langle a_{1}\right\rangle$ over $(Q, \gamma)$.

Proposition 3.2. Suppose now that $A$ is split and that $\sigma$ is an arbitrary involution over $A$. Then $(A, \sigma) \simeq\left(\mathrm{M}_{2}(K), \tau_{1}\right) \otimes_{K}\left(\mathrm{M}_{2}(K), \tau_{2}\right)$.

Proof. If $\sigma$ is orthogonal, the statement follows from $\mathrm{PC}(2)$. If $\sigma$ is symplectic, this is due to Serhir and Tignol in [17, Proposition]. Suppose now that $\sigma$ is unitary. By a theorem due to Albert (see [8, Proposition 2.22]), we can write $\left(Q_{i}, \sigma_{i}\right)=\left(D_{i}, \gamma_{i}\right) \otimes_{F}(K,-)$ where $D_{i}$ is a quaternion algebra over $F$ and $\gamma_{i}$ is its canonical involution for $i=1,2$. Then, $(A, \sigma) \simeq\left(\left(D_{1}, \gamma_{1}\right) \otimes_{F}\left(D_{2}, \gamma_{2}\right)\right) \otimes_{F}$ $(K,-)$. As $A$ is split, the index of $D_{1} \otimes_{F} D_{2}$ is at most 2 and we conclude by using Proposition 3.1 in the orthogonal case.

\subsection{Isotropic hermitian Pfister forms over multiquater- nion algebras with involution}

This Subsection is devoted to the proof of the following theorem which is a more detailed version of Theorem 1.1: 
Theorem 3.3. Let $(A, \sigma)=\left(Q_{1}, \sigma_{1}\right) \otimes_{K} \cdots \otimes_{K}\left(Q_{n}, \sigma_{n}\right)$ be a multiquaternion algebra with involution.

(1) Suppose that $r=0$. Then, any hermitian Pfister form over $(A, \sigma)$ satisfies the property $(I \Rightarrow H)$.

(2) Suppose that $r \geq 1$. If $h$ is a hermitian Pfister form over $(A, \sigma)$ which is isotropic, then $2^{r-1} \times h$ is hyperbolic.

We first need the following:

Lemma 3.4. Let $(B, \tau) \otimes_{K}(C, v)$ be a central simple algebra with involution over $K$ such that $(C, v)=\left(\mathrm{M}_{2}(K), v_{1}\right) \otimes_{K} \cdots \otimes_{K}\left(\mathrm{M}_{2}(K), v_{t}\right)$. Let $\Phi: W\left(B \otimes_{K}\right.$ $C, \tau \otimes v) \simeq W^{\eta}(B, \tau)$ be a $W\left(K,\left.\sigma\right|_{K}\right)$-module isomorphism obtained by Morita equivalence, where $\eta=1$ if $v$ is orthogonal or unitary, $\eta=-1$ if $v$ is symplectic. Let $h$ be an $n$-fold hermitian Pfister form over $(B, \tau) \otimes_{K}(C, v)$. In the case where $v$ is orthogonal or unitary, $\Phi(h)$ is similar to an $(n+t)$-fold hermitian Pfister form over $(B, \tau)$. In the case where $v$ is symplectic, $h$ is hyperbolic over $(B, \tau)$.

Proof. For the definition of $\Phi$, we refer to [7, Chapter 1, Theorem 9.3.5] or [5, Théorème 2.2.1]. Note that $\tau \otimes v$ is adjoint to the hermitian form $\langle 1\rangle$ over $(B, \tau) \otimes_{K}(C, v)$. Furthermore, $\tau \otimes v$ is isomorphic to the involution adjoint to the $\eta$-hermitian form $\Phi(\langle 1\rangle)$ over $(B, \tau)$. Suppose first that $v$ is symplectic. Then $v$ is adjoint to a skew-symmetric bilinear and hence hyperbolic form over $K$. This implies that $\Phi(\langle 1\rangle),\langle 1\rangle$ and finally $h$ are hyperbolic. Suppose now that $v$ is orthogonal or unitary. Then, for all $i=1, \cdots, t, v_{i}$ is adjoint to a 1-fold hermitian Pfister form over $\left(K,\left.\sigma\right|_{K}\right)$. Therefore $v_{1} \otimes \cdots \otimes v_{t}$ is adjoint to a $t$-fold hermitian Pfister form over $\left(K,\left.\sigma\right|_{K}\right)$. Thus $\Phi(\langle 1\rangle)$ is similar to a $t$-fold hermitian Pfister form over $(B, \tau)$ hence $\Phi(h)$ is similar to a $(n+t)$-fold hermitian Pfister form over $(B, \tau)$.

We are now ready to prove the theorem.

Proof. (1) For any division algebras $Q_{i}, Q_{j} \in\left\{Q_{1}, \cdots, Q_{n}\right\}$ with $Q_{i} \simeq Q_{j}$ with $i \neq j$, we can write $\left(Q_{i}, \sigma_{i}\right) \otimes_{K}\left(Q_{j}, \sigma_{j}\right)=\left(\mathrm{M}_{2}(K), v_{i}\right) \otimes_{K}\left(\mathrm{M}_{2}(K), v_{j}\right)$, by Proposition 3.2. It follows that $(A, \sigma) \simeq\left(\mathrm{M}_{2}(K), v_{1}\right) \otimes_{K} \cdots \otimes_{K}\left(\mathrm{M}_{2}(K), v_{n}\right)$. Let $h$ be an isotropic hermitian Pfister form over $(A, \sigma)$. We then apply Lemma 3.4 with $(B, \tau)=\left(K,\left.\sigma\right|_{K}\right)$ and $(C, v)=(A, \sigma)$. If $\sigma$ is symplectic then $h$ is hyperbolic. If $\sigma$ is orthogonal or unitary, let $\Phi: W(A, \sigma) \simeq W\left(K,\left.\sigma\right|_{K}\right)$ be as in the lemma. As $\Phi(h)$ is isotropic and is similar to a hermitian Pfister form over $\left(K,\left.\sigma\right|_{K}\right), \Phi(h)$ hence $h$ is hyperbolic.

(2) We proceed by induction on $r$. We first need the following

Claim. We claim that we can suppose that $n=r$. We may suppose that $Q_{1}, \cdots, Q_{s}$ are division and that $Q_{s+1}, \cdots, Q_{n}$ are split. Proceeding as in (1) and after reindexing, we can write $(A, \sigma) \simeq\left(Q_{1}, \sigma_{1}\right) \otimes_{K} \cdots \otimes_{K}\left(Q_{r}, \sigma_{r}\right) \otimes$ $\left(\mathrm{M}_{2}(K), v_{1}\right) \otimes_{K} \cdots \otimes_{K}\left(\mathrm{M}_{2}(K), v_{n-r}\right)$ where $Q_{1}, \cdots, Q_{r}$ are quaternion division algebras such that $Q_{i} \not Q_{j}$ for $i \neq j$. Let $h$ be an isotropic hermitian Pfister form over $(A, \sigma)$. We apply Lemma 3.4 taking $(B, \tau)=\left(Q_{1}, \sigma_{1}\right) \otimes_{K}$ $\cdots \otimes_{K}\left(Q_{r}, \sigma_{r}\right)$ and $(C, v)=\left(\mathrm{M}_{2}(K), v_{1}\right) \otimes_{K} \cdots \otimes_{K}\left(\mathrm{M}_{2}(K), v_{n-r}\right)$. Let $\Phi$ : $W(A, \sigma) \simeq W^{\eta}\left(Q_{1} \otimes_{K} \cdots \otimes_{K} Q_{r}, \sigma_{1} \otimes \cdots \otimes \sigma_{r}\right)$ be as in the lemma. As $\Phi(h)$ is an isotropic hermitian Pfister form over $\left(Q_{1}, \sigma_{1}\right) \otimes_{K} \cdots \otimes_{K}\left(Q_{r}, \sigma_{r}\right)$, this establishes the claim.

Suppose $r=1$. By the claim, we can suppose that $n=1$. If $\sigma$ is symplectic, the result is clear. If $\sigma$ is unitary or orthogonal, the result follows from Theorem 
2.5. Suppose now that $r \geq 2$. Again, by the claim, we can suppose that $n=r$. We distinguish three cases.

Suppose first that $\sigma$ is unitary. By a theorem due to Albert (see [8, Proposition 2.22]), one may write $\left(Q_{r}, \sigma_{r}\right)=\left(Q \otimes_{F} K, \gamma \otimes-\right)$ where $Q$ is a quaternion algebra over $F$ and $\gamma$ is its canonical involution. Write $Q=(a, b)_{F}$ and let $\{1, i, j, k\}$ be a quaternion basis of $Q$. Set $\lambda_{1}=i \otimes 1 \in Q_{r}, \mu_{1}=j \otimes 1 \in Q_{r}$ and $L_{1}=F\left(\lambda_{1}\right)$. Then the centralizer of $L_{1}$ in $Q_{r}$ is $L_{1} \otimes_{F} K$. Moreover, as $Q_{r}$ is division, $L_{1} \otimes_{F} K$ is a field. Now set $\lambda=1 \otimes \cdots \otimes 1 \otimes \lambda_{1}, \mu=$ $1 \otimes \cdots \otimes 1 \otimes \mu_{1} \in Q_{1} \otimes_{K} \cdots \otimes_{K} Q_{r}=A$ and $L=F(\lambda)$. The centralizer of $L$ in $A$ is $\widetilde{A}=Q_{1} \otimes_{K} \cdots \otimes Q_{r-1} \otimes_{K}\left(L_{1} \otimes_{F} K\right)$. In this case, a transfer map $\pi_{1}: W(A, \sigma) \rightarrow W\left(\widetilde{A}, \sigma_{1}\right)$ can be defined where $\sigma_{1}=\left.\sigma\right|_{\widetilde{A}}$ is unitary (more precisely, we have $A=\widetilde{A} \oplus \mu \widetilde{A}$ and $\pi_{1}$ is induced by the first projection map). Let $h=\varphi \cdot\langle 1\rangle$ be an isotropic hermitian Pfister form over $(A, \sigma)$, where $\varphi$ is a quadratic Pfister form over $F$. Then $\pi_{1}(h) \simeq \varphi \cdot\left\langle 1,-\mu^{2}\right\rangle$ is an isotropic hermitian Pfister form over $\left(\widetilde{A}, \sigma_{1}\right)$ hence $2^{r-2} \times \pi_{1}(h)=0 \in W\left(\widetilde{A}, \sigma_{1}\right)$ by induction hypothesis. We also have $\left(2^{r-2} \times \varphi\right) \cdot\left\langle 1,-\mu^{2}\right\rangle=0 \in W(A, \sigma)$ and, as $\langle 1\rangle=\left\langle-\mu^{2}\right\rangle \in W(A, \sigma)$, we finally obtain that $2^{r-1} \times h=0 \in W(A, \sigma)$.

Suppose now that at least one of the $\sigma_{i}$ 's, say $\sigma_{r}$, is symplectic. Write $Q_{r}=$ $(a, b)_{K}$ and $\{1, i, j, k\}$ be a quaternion basis of $Q_{r}$. Set $\lambda=1 \otimes \cdots \otimes 1 \otimes i, \mu=$ $1 \otimes \cdots 1 \otimes j$ and $L=K(\lambda)$. As in the previous case, $\widetilde{A}=Q_{1} \otimes_{K} \cdots \otimes_{K} Q_{r-1} \otimes L$ is the centralizer of $L$ in $A$ and we have the transfer map $\pi_{1}: W(A, \sigma) \rightarrow W\left(\widetilde{A}, \sigma_{1}\right)$ where $\sigma_{1}=\left.\sigma\right|_{\widetilde{A}}$ has unitary type. We conclude by using the previous case and similar calculations.

Last, suppose that all the $\sigma_{i}$ 's are orthogonal. Write $Q_{r}=(a, b)_{K}$ with $i^{2}=a, j^{2}=b, i j=-j i, \sigma_{r}(i)=i, \sigma_{r}(j)=-j$. Then $\gamma=\operatorname{Int}(j) \circ \sigma_{r}$ is the canonical involution of $Q_{r}$. Choose $\lambda$ and $\mu$ as in the previous case. We then have a $W(K)$-module isomorphism $W(A, \sigma) \simeq W^{-1}\left(A, \sigma_{1} \otimes \cdots \otimes \sigma_{r-1} \otimes \gamma\right)$ : $h \mapsto \mu h$. Take $\widetilde{A}$ as in the previous case and let $\tau=\sigma_{1} \otimes \cdots \otimes \sigma_{r-1} \otimes \gamma$ on $A$ and $\tau_{2}=\sigma_{1} \otimes \cdots \otimes \sigma_{r-1} \otimes \operatorname{id}_{L}$ on $\widetilde{A}$. Note that $\tau_{2}$ is orthogonal. As $A=\widetilde{A} \oplus \mu \widetilde{A}$, the second projection map induces a transfer map $\pi_{2}: W^{-1}(A, \tau) \rightarrow W\left(\widetilde{A}, \tau_{2}\right)$. If $h=\varphi \cdot\langle 1\rangle$ is an isotropic hermitian Pfister form over $(A, \sigma)$, then $\pi_{2}(\mu h)=$ $\varphi \cdot\left\langle 1,-\mu^{2}\right\rangle$ is an isotropic hermitian Pfister form over $\left(\widetilde{A}, \tau_{2}\right)$. We conclude as in the unitary case.

Remark 3.5. A part of the previous result has already been proved by the first and the third author independently (see [5, Proposition 5.2.10] and [12, Theorem 25.2]).

\subsection{About certain submodules of the Witt group of a mul- tiquaternion algebra with involution}

The first step in understanding the quadratic forms that belong to a given power of the fundamental ideal of $W(K)$ is the following result (see [9, Chapter $\mathrm{X}, \S 5]$ for more details):

Theorem 3.6 (Arason-Pfister). Let $q$ be a positive-dimensional anisotropic quadratic form over $K$. If $q \in I^{n}(K)$, then $\operatorname{dim} q \geq 2^{n}$. 
An immediate consequence of this result is the "Krull Intersection Property" for the ideals $I^{n}(K)$, that is $\bigcap_{n>0} I^{n}(K)=0$ in $W(K)$.

For an integer $n \geq 1$, denote by $I^{n}(A, \sigma)$ the $W(F)$-submodule of $W(A, \sigma)$ generated by $n$-fold hermitian Pfister forms over $(A, \sigma)$. The purpose of this Subsection is to prove a weak analogue of Theorem 3.6 for the modules $I^{n}(A, \sigma)$ in a multiquaternion algebra with involution.

Before that, we need to recall further facts and notations. Recall that the rank of a hermitian form $(V, h)$ over $(A, \sigma)$ is defined as the integer $p$ such that $V \simeq S^{p}$, where $S$ is a simple right $A$-module. The rank of $(V, h)$ over $(A, \sigma)$ will be denoted by $\operatorname{rk}_{A}(h)$. It can be seen that the rank is invariant under Morita equivalence (the proof of this fact can be adapted from [6, Lemma A.5]). Using Morita equivalence, one can also see that for every non-hyperbolic hermitian form over $(A, \sigma)$, there exists an anisotropic hermitian form $h_{\text {an }}$ over $(A, \sigma)$ which is unique up to isometry such that $[h]=\left[h_{\text {an }}\right] \in W(A, \sigma)$ (see $[6$, Corollary A.7]). The form $h_{\text {an }}$ is called the anisotropic part of $h$.

Theorem 3.7. Let $(A, \sigma)=\left(Q_{1}, \sigma_{1}\right) \otimes_{K} \cdots \otimes_{K}\left(Q_{m}, \sigma_{m}\right)$ be a multiquaternion algebra with involution. Let $h$ be a positive-dimensional anisotropic hermitian form over $(A, \sigma)$. If $h \in I^{n}(A, \sigma)$ and if $2^{r} \times h$ is not hyperbolic over $(A, \sigma)$, then $r k_{A}(h) \geq 2^{n+m-r}$.

Proof. We prove the property by induction on $r$. We may assume that $h$ is an $n$-fold hermitian Pfister form over $(A, \sigma)$. We first need to prove the following Claim. We claim that we can suppose that $m=r$. Proceeding as in the proof of Theorem 3.3(2), we write $(A, \sigma) \simeq\left(Q_{1}, \sigma_{1}\right) \otimes_{K} \cdots\left(Q_{r}, \sigma_{r}\right) \otimes_{K}\left(\mathrm{M}_{2}(K), v_{1}\right) \otimes_{K}$ $\cdots \otimes_{K}\left(\mathrm{M}_{2}(K), v_{m-r}\right)$ where $Q_{1}, \cdots, Q_{r}$ are quaternion division algebras such that $Q_{i} \not \Varangle Q_{j}$ for $i \neq j$. Applying Lemma 3.4, we can assume that $(A, \sigma)=$ $\left(Q_{1}, \sigma_{1}\right) \otimes_{K} \cdots\left(Q_{r}, \sigma_{r}\right)$ where the $Q_{i}$ 's are division algebras such that $Q_{i}$ is not isomorphic to $Q_{j}$ for $i \neq j$ and that $h$ is an $(n+m-r)$-fold hermitian Pfister form over $(A, \sigma)$ thus establishing the claim.

Suppose that $r=0$. By the claim, we can see $h$ as an $(n+m)$-fold anisotropic hermitian Pfister form over $\left(K,\left.\sigma\right|_{K}\right)$ and the property follows from Theorem 3.6. Suppose now that $r \geq 1$. Again, by the claim, we may assume that $m=r$. As in the proof of Theorem 3.3, we have to distinguish three cases. In this proof, we will only focus on the unitary case, the two other cases being similar. Let $\lambda, \mu \in A^{\times}, L$ and $\widetilde{A}$ be as in the proof of Theorem 3.3. Put $\tau_{1}=\left.\sigma\right|_{\widetilde{A}}$. In this case, we know that $A=\widetilde{A} \oplus \mu \widetilde{A}$ and there is a transfer map $\pi_{1}: W(A, \sigma) \rightarrow W\left(\widetilde{A}, \tau_{1}\right)$ induced by the first projection. Write $h=q \cdot\langle 1\rangle \in W(A, \sigma)$ such that $q$ is an $(n+m-r)$-fold quadratic Pfister form over $F$. Then $\pi_{1}(h)=q \cdot\left\langle 1,-\mu^{2}\right\rangle$ is an $(n+m-r+1)$-fold hermitian Pfister form over $\left(\widetilde{A}, \tau_{1}\right)$. The fact that $2^{r} \times h$ is not hyperbolic over $(A, \sigma)$ implies that $2^{r-1} \times \pi_{1}(h)$ is not hyperbolic over $\left(\widetilde{A}, \tau_{1}\right)$. Otherwise, as in the proof of Theorem 3.3 , we would have $0=$ $2^{r-1} \times\left(q \cdot\left\langle 1,-\mu^{2}\right\rangle\right)=2^{r} \times h \in W(A, \sigma)$. In particular, we can consider the anisotropic part $\pi_{1}(h)_{\text {an }}$ of $\pi_{1}(h)$ and $\pi_{1}(h)_{\text {an }} \in I^{n+m-r+1}\left(\widetilde{A}, \tau_{1}\right)$. By induction hypothesis, we have the following inequalities

$$
2 \times \operatorname{rk}_{A}(h) \geq \operatorname{rk}_{\widetilde{A}}\left(\pi_{1}(h)\right) \geq \operatorname{rk}_{\widetilde{A}}\left(\pi_{1}(h)_{a n}\right) \geq 2^{n+m-r+1},
$$

which lead to $\operatorname{rk}_{A}(h) \geq 2^{n+m-r}$. 
Remark 3.8. In the previous statement, suppose that $A=D$ is division. In this case, one has $r=m$, and the rank of $h$ is nothing but the dimension of the underlying $D$-vector space. The statement says that, if $h$ is anisotropic with $h \in I^{n}(D, \sigma)$ and if $2^{r} \times h$ is not hyperbolic over $(D, \sigma)$ then $\operatorname{dim} h \geq 2^{n}$. In this manner, we see that Theorem 3.7 is a weak analogue of Theorem 3.6 for the considered submodules.

As an immediate Corollary, we get:

Corollary 3.9. Let $(A, \sigma)=\left(Q_{1}, \sigma_{1}\right) \otimes_{K} \cdots \otimes_{K}\left(Q_{m}, \sigma_{m}\right)$ be a multiquaternion algebra with involution. Then $\bigcap_{n \geq 0} I^{n}(A, \sigma)$ is a $2^{r}$-torsion submodule of $W(A, \sigma)$.

\subsection{Consequences for Pfister involutions}

In this Subsection, we draw some consequences of Theorem 3.3 for multiquaternion algebras with involution. We are particularly interested in Pfister involutions.

We first want to talk about the weak hyperbolicity of these involutions. Recall that, for any central simple algebra with involution $(A, \sigma)$, the $n$-fold orthogonal sum $\boxplus^{n}(A, \sigma)$ is defined by $\boxplus^{n}(A, \sigma)=\left(M_{n}(K), *\right) \otimes_{K}(A, \sigma)$ where $*$ is the conjugate transpose involution. An algebra with involution $(A, \sigma)$ is then said to be weakly hyperbolic if there is an integer $n$ such that $\boxplus^{n}(A, \sigma)$ is hyperbolic. This notion has been defined by Unger in [19] (see also [11]). We refer to $[8, \S 6]$ for basic notions and properties about isotropic and hyperbolic involutions.

Corollary 3.10. Suppose that $(A, \sigma)$ is a multiquaternion algebra with involution. Then, if $(A, \sigma)$ is isotropic, it is weakly hyperbolic. More precisely, if $(A, \sigma)$ is isotropic, $\boxplus^{m}(A, \sigma)$ is hyperbolic where $m=\max \left(1,2^{r-1}\right)$.

Proof. If $r=0$, the result is clear by Theorem 3.3(1). Otherwise, $\sigma$ is nothing but the involution which is adjoint to the hermitian form $\langle 1\rangle$ over $(A, \sigma)$. By Theorem $3.3(2), 2^{r-1} \times\langle 1\rangle$ is hyperbolic whence the corollary.

Now, we would like to state consequences of Theorem 3.3 for linked fields. We begin by a general statement.

Theorem 3.11. Let $n$ be a non-zero positive integer. Suppose that, for any multiquaternion algebra with orthogonal involution $(A, \sigma)$ of degree $2^{n}$, the property $(I \Rightarrow H)$ holds for the hermitian form $\langle 1\rangle$ over $\left(A \otimes_{K} L, \sigma \otimes \mathrm{id}_{\mathrm{L}}\right)$ for any field extension $L / K$. Then $\mathrm{PC}(n)$ holds.

Proof. Suppose that $A$ is split. By Morita equivalence, we have a $W(K)$ module isomorphism $\Phi: W(A, \sigma) \simeq W(K)$. Let $q=\Phi(\langle 1\rangle)$. We show that $q$ is similar to a Pfister form over $K$. Let $L$ be an extension of $K$ such that $q_{L}$ is isotropic. Write $A_{L}$ for $A \otimes_{K} L$ and $\sigma_{L}$ for $\sigma \otimes \mathrm{id}_{L}$. We can find a commutative diagram

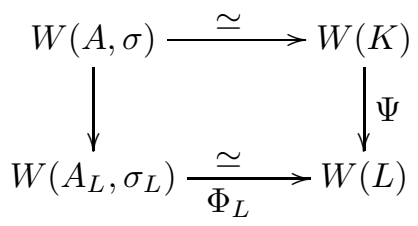


where the horizontal maps are Morita equivalences, the vertical maps are scalar extensions to $L$ and where $\Psi(q)=q_{L}$. Then $\Psi(q)=\Phi_{L}(\langle 1\rangle)$ is isotropic, hence hyperbolic by hypothesis. This shows that $q$ is similar to a Pfister form over $K$ and that $\mathrm{PC}(n)$ holds.

In the case of linked fields, Theorem 3.3 has a particularly nice statement:

Theorem 3.12. Assume that $K$ is a linked field. Then the property $(I \Rightarrow$ $H$ ) holds for any hermitian Pfister form over any multiquaternion algebra with involution of the first kind.

Proof. It follows from Theorem 3.3.

Combining the two previous results, we get the following one which has already been proved (in different ways) by Shapiro in [18, Corollary 9.13] and by Serhir and Tignol in [17, Theorem]:

Corollary 3.13. Assume that $K$ is a linked field. Then, Conjecture 2.8 holds.

\section{The invariant $u_{\alpha}$}

In this section, we will always assume that $Q$ is a quaternion division algebra over $K$ endowed with its canonical involution $\gamma$.

\subsection{The invariant $u_{\alpha}$}

Let us fix further notations. We denote by $\operatorname{Skew}_{1}(Q, \gamma)$ the set of isometry classes of non-degenerate 1-dimensional skew-hermitian forms over $(Q, \gamma)$ and, for any $a \in K^{\times}$, we put

$$
H_{a}(Q, \gamma)=\left\{h \in \operatorname{Skew}_{1}(Q, \gamma) \mid \operatorname{disc} h=a\right\} .
$$

If $(E, \sigma)$ is a commutative field with involution we denote $\Sigma(E, \sigma)$ the set $\left\{\sigma\left(d_{1}\right) d_{1}+\cdots+\sigma\left(d_{n}\right) d_{n} \mid d_{i} \in E, n \geqslant 1\right\}$ and $s(E, \sigma)$ the level of $(E, \sigma)$, that is $s(E, \sigma)=\sup \{n \in \mathbb{N} \mid n \times\langle 1\rangle$ is anisotropic over $(E, \sigma)\} \in \mathbb{N} \cup\{\infty\}$. If $(V, h)$ is a skew-hermitian form over $(Q, \gamma)$, we write $\Delta(h)=\{h(x, x) \mid x \in V \backslash\{0\}\}$.

Let $\alpha \in Q^{\times}$be a pure quaternion. Let $G_{\alpha}$ denote the subgroup of $K^{\times}$ defined by

$$
G_{\alpha}=\left\{c \in K^{\times} \mid\langle c \alpha\rangle \simeq\langle\alpha\rangle \text { as skew-hermitian forms over }(Q, \gamma)\right\} .
$$

Let $\beta \in Q^{\times}$be a pure quaternion anticommuting with $\alpha$ and $b=\beta^{2} \in K^{\times}$. Denote by $N(K(\alpha))$ the norm group of the quadratic extension $K(\alpha) / K$. According to a lemma due to Scharlau, for any $c \in K^{\times}, c \in G_{\alpha}$ if and only if $c$ is represented by one of the quadratic forms $\langle 1,-a\rangle$ and $\langle b,-a b\rangle$ (see [14, Ch. 10, $3.4]$ ). In other words, we have

$$
G_{\alpha}=N\left(K(\alpha)^{\times}\right) \cup b N\left(K(\alpha)^{\times}\right) .
$$

It follows that $N\left(K(\alpha)^{\times}\right)$is a subgroup of index 2 in $G_{\alpha}$ and that $\left|K^{\times} / G_{\alpha}\right|=$ $\frac{1}{2}\left|K^{\times} / N\left(K(\alpha)^{\times}\right)\right|$, in the case where $K^{\times} / N\left(K(\alpha)^{\times}\right)$is a finite group. 
Proposition 4.1. Let $\alpha \in Q^{\times}$be a pure quaternion and let $a=\operatorname{Nrd}_{Q / K}(\alpha)$. Then there is a bijection between the sets $H_{a}(Q, \gamma)$ and $K^{\times} / G_{\alpha}$.

Proof. By [3, Proposition 5.1], $H_{a}(Q, \gamma)$ is the set of isometry classes of 1-dimensional skew-hermitian forms $\langle c \alpha\rangle$ for $c \in K^{\times}$. We then easily see that the map $\langle c \alpha\rangle \mapsto c G_{\alpha}$ induces a bijection from $H_{a}(Q, \gamma)$ to $K^{\times} / G_{\alpha}$.

In the sequel, we will need the following technical lemma.

Lemma 4.2. Let $\psi$ be a skew-hermitian form over $(Q, \gamma)$ and let $\alpha \in Q^{\times}$ be a pure quaternion. Let $E_{\alpha}$ be the centralizer of $K(\alpha)$ in $Q$. Suppose that $s\left(E_{\alpha},\left.\gamma\right|_{E_{\alpha}}\right)<\infty$. If $\varphi=\psi \perp\langle\alpha\rangle$ is anisotropic then

$$
\Delta(\psi) \cap \alpha \Sigma\left(E_{\alpha},\left.\gamma\right|_{E_{\alpha}}\right) \subsetneq \Delta(\varphi) \cap \alpha \Sigma\left(E_{\alpha},\left.\gamma\right|_{E_{\alpha}}\right) .
$$

Proof. We write $0=\gamma\left(d_{0}\right) d_{0}+\cdots+\gamma\left(d_{s}\right) d_{s}$ where $s=s\left(E_{\alpha},\left.\gamma\right|_{E_{\alpha}}\right)$ and $d_{0}, \cdots, d_{s} \in E_{\alpha}{ }^{\times}$. We always have $\Delta(\psi) \cap \alpha \Sigma\left(E_{\alpha},\left.\gamma\right|_{E_{\alpha}}\right) \subseteq \Delta(\varphi) \cap \alpha \Sigma\left(E_{\alpha},\left.\gamma\right|_{E_{\alpha}}\right)$. Suppose that $\Delta(\psi) \cap \alpha \Sigma\left(E_{\alpha},\left.\gamma\right|_{E_{\alpha}}\right)=\Delta(\varphi) \cap \alpha \Sigma\left(E_{\alpha},\left.\gamma\right|_{E_{\alpha}}\right)$. In this situation, we claim that $\alpha\left(\gamma\left(d_{0}\right) d_{0}+\cdots+\gamma\left(d_{i}\right) d_{i}\right) \in \Delta(\varphi) \cap \alpha \Sigma\left(E_{\alpha},\left.\gamma\right|_{E_{\alpha}}\right)$ for $i=0, \cdots, s$. For $i=s$ this implies that $\varphi$ is isotropic which is a contradiction. For $i=0$ the claim follows from the fact that $\alpha\left(\gamma\left(d_{0}\right) d_{0}\right)=\gamma\left(d_{0}\right) \alpha d_{0} \in \Delta(\varphi) \cap \alpha \Sigma\left(E_{\alpha},\left.\gamma\right|_{E_{\alpha}}\right)$. Now, if we assume that $\alpha\left(\gamma\left(d_{0}\right) d_{0}+\cdots+\gamma\left(d_{i-1}\right) d_{i-1}\right) \in \Delta(\varphi) \cap \alpha \Sigma\left(E_{\alpha},\left.\gamma\right|_{E_{\alpha}}\right)=$ $\Delta(\psi) \cap \alpha \Sigma\left(E_{\alpha},\left.\gamma\right|_{E_{\alpha}}\right)$, we readily obtain that $\alpha\left(\gamma\left(d_{0}\right) d_{0}+\cdots+\gamma\left(d_{i-1}\right) d_{i-1}\right)+$ $\alpha \gamma\left(d_{i}\right) d_{i} \in \Delta(\varphi) \cap \alpha \Sigma\left(E_{\alpha},\left.\gamma\right|_{E_{\alpha}}\right)$.

Let $\varepsilon= \pm 1$. Denote by $\operatorname{Sym}^{\varepsilon}(Q, \gamma)$ the set of $\varepsilon$-symmetric elements of $Q$ with respect to $\gamma$, that is the elements $\alpha$ of $Q$ such that $\gamma(\alpha)=\varepsilon \alpha$.

Definition 4.3. Let $\alpha \in Q^{\times}$be an $\varepsilon$-symmetric element. We define the invariant $u_{\alpha}(K)$ to be the supremum over the dimension of all quadratic forms $\varphi$ over $K$ such that the form $\varphi \cdot\langle\alpha\rangle$, as an $\varepsilon$-hermitian form over $(Q, \gamma)$, is anisotropic.

It is clear from the definition that $u_{\alpha}(K) \leqslant u(K)$. The following example shows that these two invariants can be equal or different and that the invariant $u_{\alpha}$ can be finite or infinite.

Example 4.4. Take $K=\mathbb{R}$ and $Q$ to be Hamilton's quaternion algebra endowed with its canonical involution. Then $u(K)=\infty=u_{\alpha}(K)$ for any $\alpha \in \operatorname{Sym}^{1}(Q, \gamma)$. If $\alpha \in \operatorname{Sym}^{-1}(Q, \gamma)$, it is easy to see that $u_{\alpha}(K)=1$.

\subsection{Upper bounds for the invariant $u_{\alpha}$}

Let $\alpha \in \operatorname{Sym}^{1}(Q, \gamma)$. If $u_{\alpha}(K)=\infty$, then $u(K)=u_{\alpha}(K)=\infty$. If $u_{\alpha}(K)$ is finite it is easily seen, via the usual injection $W(Q, \gamma) \hookrightarrow W(K)$, that $4 u_{\alpha}(K) \leq$ $u(K)$.

¿From now on, we suppose that $\alpha$ is a skew-symmetric element in $Q^{\times}$with respect to its canonical involution $\gamma$ and we write $a=\operatorname{Nrd}_{Q / K}(\alpha)$.

In this situation, we obtain an analogue of Kneser's result mentioned in the Introduction.

Proposition 4.5. Suppose that $K$ is a non-real field. Then $u_{\alpha}(K) \leqslant\left|K^{\times} / G_{\alpha}\right|$. 
Proof. Let $\varphi \cdot\langle\alpha\rangle$ be an anisotropic skew-hermitian form over $(Q, \gamma)$ where $\varphi=$ $\left\langle a_{1}, \cdots, a_{n}\right\rangle$ is a quadratic form over $K, a_{i} \in K^{\times}$. Denote $h_{i}=\left\langle a_{1}, \cdots, a_{i}\right\rangle \cdot\langle\alpha\rangle$. According to Lemma 4.2,

$$
\Delta\left(h_{i}\right) \cap \alpha_{i+1} \Sigma\left(K(\alpha),\left.\gamma\right|_{K(\alpha)}\right) \subsetneq \Delta\left(h_{i+1}\right) \cap \alpha_{i+1} \Sigma\left(K(\alpha),\left.\gamma\right|_{K(\alpha)}\right),
$$

where $\alpha_{i+1}=a_{i+1} \alpha$. As $K$ is non-real, we have $\Sigma\left(K(\alpha),\left.\gamma\right|_{K(\alpha)}\right)=K$. Consequently for every $i$ we have $\alpha_{i+1} \Sigma\left(K(\alpha),\left.\gamma\right|_{K(\alpha)}\right)=\alpha \cdot K$. Therefore we have a sequence of inclusions

$$
\Delta\left(h_{1}\right) \cap K \cdot \alpha \subsetneq \Delta\left(h_{2}\right) \cap K \cdot \alpha \subsetneq \cdots \subsetneq \Delta\left(h_{n}\right) \cap K \cdot \alpha \subset H_{a}(Q, \gamma) .
$$

We conclude that $\left|H_{a}(Q, \gamma)\right| \geqslant n$. Thus $u_{\alpha}(K) \leqslant\left|H_{a}(Q, \gamma)\right|=\left|K^{\times} / G_{\alpha}\right|$ by Proposition 4.1 .

Remark 4.6. It follows from the proof that the condition $s\left(K(\alpha),\left.\gamma\right|_{K(\alpha)}\right)<\infty$ is actually sufficient to have the conclusion of the previous Proposition.

We now come to the proof of Theorem 1.3:

Theorem. Suppose that $s\left(K(\alpha),\left.\gamma\right|_{K(\alpha)}\right) \leqslant 2$. Then, either $u_{\alpha}(K)=\left|K^{\times} / G_{\alpha}\right|$ or $u_{\alpha}(K) \leqslant \frac{1}{2}\left|K^{\times} / G_{\alpha}\right|$.

We first need the following:

Lemma 4.7. Suppose that $u_{\alpha}(K)<\left|K^{\times} / G_{\alpha}\right|<\infty$ and that $\varphi$ is a quadratic form over $K$. If $\varphi$ has a diagonalization such that it has more than $\left|K^{\times} / G_{\alpha}\right| / 2$ distinct entries modulo the group $G_{\alpha}$, then $\varphi \cdot\langle\alpha\rangle$ is isotropic over $(Q, \gamma)$.

Proof. We can naturally consider $K^{\times} / G_{\alpha}$ as a $\mathbb{Z} / 2 \mathbb{Z}$-vector space. Let $\left\{x_{1}, \cdots, x_{r}\right\}$ be a basis of this vector space. Let $\ell=\left|K^{\times} / G_{\alpha}\right|$. The $\ell$-dimensional skew-hermitian form $h=\left\langle\left\langle x_{1}, \cdots, x_{r}\right\rangle\right\rangle \cdot\langle\alpha\rangle$ is isotropic over $(Q, \gamma)$ because $\ell>u_{\alpha}(K)$. It follows from Theorem 2.6 that $h$ is hyperbolic.

Now let $\varphi$ be any quadratic form which has a diagonalization consisting in distinct entries modulo $G_{\alpha}$. In particular $\varphi \cdot\langle\alpha\rangle$ is a subform of the hyperbolic form $h$. Consequently if $\operatorname{dim} \varphi>\ell / 2, \varphi \cdot\langle\alpha\rangle$ must be isotropic.

We are now ready to prove Theorem 1.3:

Proof. We may assume that $\left|K^{\times} / G_{\alpha}\right|<\infty$. We already know that $u_{\alpha}(K) \leqslant\left|K^{\times} / G_{\alpha}\right|$ (see Remark 4.6), so assume that $u_{\alpha}(K)<\left|K^{\times} / G_{\alpha}\right|$. If $s\left(K(\alpha),\left.\gamma\right|_{K(\alpha)}\right)=1$ then for every quadratic form $\varphi$ which has two entries which are equal modulo $G_{\alpha}$ in some diagonalization, the form $\varphi \cdot\langle\alpha\rangle$ is isotropic over $(Q, \gamma)$. Indeed, the subform of $\varphi \cdot\langle\alpha\rangle$ given by these two coefficients is already defined and isotropic over $\left(K(\alpha),\left.\gamma\right|_{K(\alpha)}\right)$. Thus that if $\varphi \cdot\langle\alpha\rangle$ is anisotropic over $(Q, \gamma)$ then all its entries are distinct modulo $G_{\alpha}$. It follows from Lemma 4.7 that $u_{\alpha}(K) \leqslant \frac{1}{2}\left|K^{\times} / G_{\alpha}\right|$.

So let assume that $s\left(K(\alpha),\left.\gamma\right|_{K(\alpha)}\right)=2$. We consider two cases: $-1 \in G_{\alpha}$ and $-1 \notin G_{\alpha}$.

If $-1 \in G_{\alpha}$ then $\langle\alpha\rangle \simeq\langle-\alpha\rangle$ as skew-hermitian forms over $(Q, \gamma)$. Let $\varphi$ be a quadratic form over $K$. If $\varphi$ has more than two equal entries modulo $G_{\alpha}$ in some diagonalization, say $\varphi \simeq\langle a, b, \cdots\rangle$ where $a=b \bmod G_{\alpha}$, then $\varphi \cdot\langle\alpha\rangle \simeq\langle a, a, \cdots\rangle \cdot\langle\alpha\rangle \simeq\langle a,-a, \cdots\rangle \cdot\langle\alpha\rangle$, so $\varphi \cdot\langle\alpha\rangle$ is isotropic. It follows 
that, if $\varphi \cdot\langle\alpha\rangle$ is anisotropic, then all its entries are distinct modulo $G_{\alpha}$. Now, Proposition 4.7 implies that $u_{\alpha}(K) \leqslant \frac{1}{2}\left|H_{a}(Q, \gamma)\right|$.

Assume that $-1 \notin G_{\alpha}$. Let $\left\{-1, a_{2}, \cdots, a_{r}\right\}$ be a $\mathbb{Z} / 2 \mathbb{Z}$-basis for $K^{\times} / G_{\alpha}$ and let $\ell=\left|K^{\times} / G_{\alpha}\right|$. Then the $\ell$-dimensional form $h=\left\langle\left\langle 1, a_{2}, \cdots, a_{r}\right\rangle\right\rangle \otimes\langle\alpha\rangle$ is isotropic over $(Q, \gamma)$ as $u_{\alpha}(K)<\ell$. By Theorem 2.6, $h$ is hyperbolic. Let $\varphi$ be a quadratic form over $K$ such that $\varphi \cdot\langle\alpha\rangle$ is anisotropic. We are going to show that $\operatorname{dim} \varphi \leqslant \frac{\ell}{2}$ which completes the proof.

First note, that in every diagonalization of $\varphi$, every entry repeats at most 2 times modulo $G_{\alpha}$ otherwise $\varphi \cdot\langle\alpha\rangle$ contains a subform isometric to $\langle a, a, a\rangle \cdot\langle\alpha\rangle$ for some $a \in K^{\times}$which is isotropic because $s\left(K(\alpha),\left.\gamma\right|_{K(\alpha)}\right)=2$.

We may now write $\varphi \simeq 2\left\langle x_{1}\right\rangle \perp \cdots \perp 2\left\langle x_{m}\right\rangle \perp\left\langle x_{m+1}, \cdots, x_{n}\right\rangle$ where $x_{i} \neq x_{j}$ modulo $G_{\alpha}$ for $i \neq j$. We also have $x_{i} \neq-x_{j}$ modulo $G_{\alpha}$ for $i \neq j$ because $\varphi \cdot\langle\alpha\rangle$ is anisotropic. So we may assume that $x_{i} \neq \pm x_{j}$ modulo $G_{\alpha}$ for $i \neq j$.

As already mentioned for any $a \in K^{\times}$the form $\langle a, a, a\rangle \cdot\langle\alpha\rangle$ is isotropic. Using Theorem 2.6, we obtain

$$
\langle a, a\rangle \cdot\langle\alpha\rangle \simeq\langle-a,-a\rangle \cdot\langle\alpha\rangle .
$$

Let $S$ be the $\mathbb{Z} / 2 \mathbb{Z}$-subspace of $K^{\times} / G_{\alpha}$ generated by $a_{2}, \cdots, a_{r}$. We have $K^{\times} / G_{\alpha}=-S \cup S$. The definition of the form $h$ implies that $h=\perp_{s \in S}\langle s, s\rangle \cdot\langle\alpha\rangle$. For every $i, 1 \leqslant i \leqslant n$ we have either $x_{i} \in S$ or $x_{i} \in-S$. In the first case, we have $2\left\langle x_{i}\right\rangle \cdot\langle\alpha\rangle \subset h$. In the second case, using (1), we obtain $2\left\langle x_{i}\right\rangle \cdot\langle\alpha\rangle \simeq$ $2\left\langle-x_{i}\right\rangle \cdot\langle\alpha\rangle \subset h$.

Since $x_{i} \neq \pm x_{j}$ modulo $G_{\alpha}$ for $i \neq j$ we conclude that $2\left\langle x_{1}, \cdots, x_{n}\right\rangle \cdot\langle\alpha\rangle$ is isometric to a subform of $h$. Therefore $\varphi \cdot\langle\alpha\rangle$ is isometric to a subform of $h$. Since $h$ is hyperbolic and $\varphi \cdot\langle\alpha\rangle$ is anisotropic, we obtain $\operatorname{dim} \varphi \leqslant \frac{1}{2} \operatorname{dim} h=\ell / 2$.

\section{Acknowledgement}

The authors are grateful to Detlev Hoffmann, David Lewis and Jean-Pierre Tignol for several valuable remarks. The first and the second named authors acknowledge the financial support provided by the European RTN Network "Algebraic K-Theory Linear Algebraic Groups, and Related Structures" (HPRNCT-2000-00287). The third named author acknowledges the financial support provided by the Research Council of Sharif University of Technology.

\section{References}

[1] E. Bayer-Fluckiger, R. Parimala, and A. Quéguiner-Mathieu. Pfister involutions. Proc. Indian Acad. Sci. Math. Sci., 113(4) (2003) 365-377.

[2] K. J. Becher. A proof of the Pfister Factor Conjecture. Preprint, Linear Algebraic Groups Preprint Server, 245 (2007).

[3] K. J. Becher, M. G. Mahmoudi. On the hermitian $u$-invariant of a quaternion algebra. Preprint, Linear Algebraic Groups Preprint Server, 188 (2005) . 
[4] R. Elman, T. Y. Lam. Quadratic forms and the $u$-invariant, I. Math. Z. 131 (1973) 283-304.

[5] N. Grenier-Boley. Groupe de Witt d'une algèbre simple centrale à involution, Ph.D. Thesis (2004).

[6] N. Grenier-Boley, M. G. Mahmoudi. Exact sequences of Witt groups Comm. Algebra 33(4) (2005) 965-986.

[7] M.-A. Knus. Quadratic and Hermitian forms over rings, Grundlehren der Mathematischen Wissenschaften, vol. 294. Springer-Verlag, Berlin 1991.

[8] M.-A. Knus, A. Merkurjev, M. Rost, and J.-P. Tignol. The book of involutions, volume 44 of American Mathematical Society Colloquium Publications. American Mathematical Society, Providence, RI, 1998.

[9] T.-Y. Lam. Introduction to quadratic forms over fields. Graduate Studies in Mathematics, 67. American Mathematical Society, Providence, RI, 2005.

[10] D. W. Lewis. Sums of hermitian squares. J. Algebra 115, no. 2 (1988) 466-480.

[11] D. W. Lewis, T. Unger. A local-global principle for algebras with involution and Hermitian forms. Math. Z. 244, no. 3 (2003) 469-477.

[12] M. G. Mahmoudi. Involutions d'une algèbre de Clifford et isotropie des formes quadratiques et hermitiennes, Ph.D. Thesis (2004).

[13] R. Parimala, R. Sridharan, V. Suresh. Hermitian analogue of a theorem of Springer. J. Algebra 243, no. 2 (2001) 780-789.

[14] W. Scharlau. Quadratic and Hermitian forms. Grundlehren der Mathematischen Wissenschaften, 270. Springer-Verlag, Berlin, 1985.

[15] A. Serhir. Niveau hermitien de certaines algèbres de quaternions. Comm. Algebra 25(8) (1997) 2531-2538.

[16] A. Serhir. Niveau hermitien d'une algèbre de quaternions à division munie d'une involution de seconde espèce. Comm. Algebra 30(10) (2002) 46214627.

[17] A. Serhir, J.-P. Tignol. The discriminant of a decomposable symplectic involution. J. Algebra 273, no. 2 (2004) 601-607.

[18] D. B. Shapiro. Compositions of quadratic forms. de Gruyter Expositions in Mathematics, 33, Walter de Gruyter, Berlin, New-York, 2000.

[19] T. Unger. Clifford algebra periodicity for central simple algebras with an involution. Comm. Algebra 29 (2001) 1141-1152.

[20] A. R. Wadsworth, D. B. Shapiro. Spaces of similarities. III. Function fields, Journal of Algebra 46 (1977) 182-188. 
Nicolas Grenier-Boley,

Laboratoire Analyse, Géométrie et Applications, UMR 7539, Institut Galilée, Université Paris 13, 99 avenue J.-B. Clément, 93430 Villetaneuse, France. Email address: grenier@math.univ-paris13.fr

Emmanuel Lequeu,

Laboratoire de Mathématiques et Applications, Pôle Sciences et Technologies, Université de La Rochelle, Avenue Michel Crépeau, 17042 La Rochelle Cedex, France. E-mail address: emmanuel.lequeu@univ-lr.fr

Mohammad Gholamzadeh Mahmoudi,

Department of Mathematical Sciences, Sharif University of Technology, P.O. Box: 11155-9415, Tehran, Iran. E-Mail address: mmahmoudi@sharif.ir 